Journal of

Applied

Crystallography

ISSN 1600-5767

\title{
PolaBer: a program to calculate and visualize distributed atomic polarizabilities based on electron density partitioning
}

\author{
Anna Krawczuk, Daniel Pérez and Piero Macchi
}

J. Appl. Cryst. (2014). 47, 1452-1458

Copyright (C) International Union of Crystallography

Author(s) of this paper may load this reprint on their own web site or institutional repository provided that this cover page is retained. Republication of this article or its storage in electronic databases other than as specified above is not permitted without prior permission in writing from the IUCr.

For further information see http://journals.iucr.org/services/authorrights.html

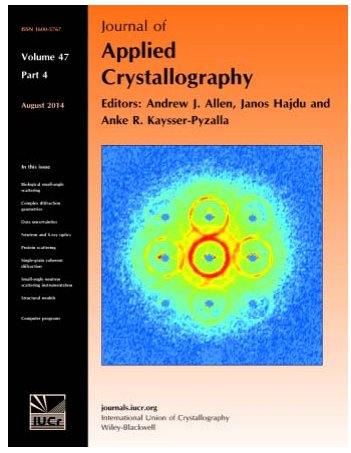

Many research topics in condensed matter research, materials science and the life sciences make use of crystallographic methods to study crystalline and non-crystalline matter with neutrons, X-rays and electrons. Articles published in the Journal of Applied Crystallography focus on these methods and their use in identifying structural and diffusioncontrolled phase transformations, structure-property relationships, structural changes of defects, interfaces and surfaces, etc. Developments of instrumentation and crystallographic apparatus, theory and interpretation, numerical analysis and other related subjects are also covered. The journal is the primary place where crystallographic computer program information is published.

Crystallography Journals Online is available from journals.iucr.org 


\author{
Journal of \\ Applied \\ Crystallography \\ ISSN 1600-5767
}

Received 30 January 2014

Accepted 12 May 2014

(C) 2014 International Union of Crystallography

\author{
Anna Krawczuk, ${ }^{a *}$ Daniel Pérez $^{\mathbf{b}}$ and Piero Macchi ${ }^{\mathbf{b} *}$ \\ ${ }^{\mathbf{a}}$ Faculty of Chemistry, Jagiellonian University, Ingardena 3, Krakow 30-060, Poland, and ${ }^{\mathbf{b}}$ Department of \\ Chemistry and Biochemistry, University of Bern, Freiestrasse 3, Bern 3012, Switzerland. Correspondence e-mail: \\ krawczuk@chemia.uj.edu.pl, piero.macchi@dcb.unibe.ch
}

\begin{abstract}
This paper describes the program PolaBer, which calculates atomic polarizability tensors from electric field perturbations of a partitioned electron density distribution. Among many possible partitioning schemes, PolaBer is currently using the quantum theory of atoms in molecules and it is interfaced to programs that apply such a partitioning. The calculation of the atomic tensors follows the idea suggested by Keith [The Quantum Theory of Atoms in Molecules: From Solid State to DNA and Drug Design, (2007), edited by C. F. Matta \& R. J. Boyd. Weinheim: Wiley-VCH], which enables the removal of the intrinsic origin dependence of the atomic charge contributions to the molecular dipole moment. This scheme allows the export, within chemically equivalent functional groups, of properties calculated from atomic dipoles, such as for example the atomic polarizabilities. The software permits visualization of the tensors and calculation of straightforward optical properties of a molecule (like the molar refractive index) or a crystal (assuming the molecule in a given crystal lattice).
\end{abstract}

\section{PolaBer: a program to calculate and visualize
distributed atomic polarizabilities based on electron \\ PolaBer: a program to calculate and visualize
distributed atomic polarizabilities based on electron density partitioning}

\section{Introduction}

The response of an electron density distribution to perturbation is a very important factor if we are to understand the behavior of molecules or solids during chemical reactions or phase transformations, solvation or molecular recognition processes, or emission of optical or spectroscopic signals.

If the perturbation is due to an external electric field, the molecular response is controlled by the (hyper)polarizability of the molecule. Quantum chemistry allows us to calculate dipolar (hyper)polarizabilities of molecules and crystals by derivation of the electronic energy $E$ with respect to the electric field $F$. For example, the $\alpha_{i j}$ component of the first-order polarizability tensor is defined as

$$
\alpha_{i j}=-\frac{\partial^{2} E}{\partial F_{i} \partial F_{j}} .
$$

Because the derivative of energy with respect to the field corresponds to the dipole moment, $\alpha_{i j}$ can be calculated also as the derivative of the dipolar moment with respect to the field:

$$
\alpha_{i j}=-\frac{\partial^{2} E}{\partial F_{i} \partial F_{j}}=\frac{\partial \mu_{j}}{\partial F_{i}} .
$$

This means that the polarizabilities are more directly connected to an easy observable like the electron density (more precisely to the moments of the electron density) rather than to the electronic energy, a quantum mechanical observable that is, however, not easily available from experiments.

Similarly to the charge distribution, a chemist would prefer to analyze the atomic and bond polarizabilities of a system rather than the total molecular quantity. There are many reasons for this. First of all, atoms or functional groups of atoms represent the way in which molecular chemists normally 'reduce' a molecule (or a molecular crystal) to rationalize the chemical functions, such as reactivity, molecular recognition, solvation etc. The same could be valid also in materials science for engineering purposes. In fact, a given property may originate from a particular functional group of the molecule, and therefore it is important to know the actual effect of each functional group. In addition, atomic parameterization is a prerequisite for semiempirical (force-field-based) modeling of intermolecular interactions, for example in molecular mechanics or molecular dynamics simulations. In this respect, transportable atomic polarizabilities are extremely useful, in analogy with transportable atomic multipolar moments (Pichon-Pesme et al., 1995; Volkov et al., 2004; Dittrich et al., 2006; Zarychta et al., 2007).

The breakdown of molecular dipole moment into atomic terms allows the contribution of each atom to the molecular polarizability to be calculated. Many atomic partitioning schemes have been proposed in the literature. Most of them were intended to distribute the molecular electric moments in terms of the constituent atoms (Stewart et al., 1975; Stewart, 1976; Hansen \& Coppens, 1978; Hirshfeld, 1977; Kurki-Suonio, 1968; Kurki-Suonio, 1977). Stone (1985) and Le Sueur \& Stone (1993) directly analyzed several partitioning schemes of the molecular polarizabilities and concluded that space-partitioned atomic polarizability volumes would be the most efficient. Bader et al. (1987), Laidig \& Bader (1990), Bader (1989) and Bader et al. (1992) also proposed hard-space partitioning of the molecular polarizabilities, based on the quantum theory of atoms in molecules (QTAIM), which was later generalized by Keith (2007). Hättig et al. (1996) first proposed the atomic partitioning of frequency-dependent polarizabilities, based on QTAIM as well as on Stone's approaches. Their main purpose was to estimate atom-atom dispersion coefficients for the evaluation of intermolecular interac- 
tion energies. Gough et al. (1996) have used QTAIM polarizabilities to compute intensities of Raman spectra. However, the results of atomic partitioning are not included in that work. Devarajan \& Glazer (1986) and Glazer \& Stadnicka (1986) have derived a theoretical background to connect anisotropic atomic polarizabilities to some optical properties, like refractive indices and optical rotatory power. In those papers, atomic polarizabilities in crystals were visualized for the first time.

We have recently shown (Krawczuk et al., 2011) that the partitioning proposed by Keith, and implemented in AIMAll (Keith, 2013), could be used to solve the problem of origin dependence that affects the partitioning of molecular (hyper)polarizabilities. The problem arises from atomic charges that produce a dipole moment when shifted from the center of charge of the molecule. It should be recalled at this point that the total molecular dipole is origin independent if the total molecular charge is zero. However, as each atom in the molecule is not neutral (apart from trivial exceptions), the charge-distance vectors generate an 'apparent' origin dependence, which is a 'true' one if the molecule is not neutral. Keith proposed to 'cancel' the atomic charges, translating them into bonds and creating, at each atom center, additional dipolar terms originated from the bond charge translation, also defined as 'directed bond contributions to the atomic charge' by Keith (2007). After this transformation, each atom possesses only dipolar terms: the electric dipole moment calculated from the uneven distribution of charge inside the atomic basin (atomic polarization) and the charge translation dipole (the sum of all bond charge translation dipoles). The molecular dipole moment is the exact sum of all these atomic terms and the origindependent charge-distance contribution has disappeared. Even for non-neutral molecules, the dipole remains invariant under a change of coordinate system, if using the same space partitioning of the electron density. The most important consequence of this operation is that the atomic polarizability terms are easier to compute and much more transportable from one atom to a chemically equivalent one belonging to another molecule (even of completely different geometry), which is an important condition for force-field approaches in molecular modeling. At the same time, this approach allows a breakdown into atomic components of the terms that give origin to optical properties of a molecule or a crystal, which is important for the design of molecular-based materials.

In the following, we will briefly review the theoretical background and present the software PolaBer, which calculates and visualizes atomic polarizability tensors.

\section{Theoretical background}

According to the QTAIM partitioning method, atomic properties such as atomic charges $Q(\Omega)$, energies $E(\Omega)$ or, in particular, dipole moments $\boldsymbol{\mu}(\Omega)$ can be calculated by integrating their corresponding operators over the volume of the atomic basin $\Omega$. The atomic dipole moment can be then defined as

$$
\boldsymbol{\mu}(\Omega)=-\int_{\Omega}\left(\mathbf{r}-\mathbf{R}_{\Omega}\right) \rho(\mathbf{r}) \mathrm{d} \mathbf{r}+\sum_{\Omega^{\prime}}\left[\mathbf{R}_{\Omega}-\mathbf{R}_{\mathrm{b}}\left(\Omega \mid \Omega^{\prime}\right)\right] Q\left(\Omega \mid \Omega^{\prime}\right),
$$

where $Q\left(\Omega \mid \Omega^{\prime}\right)$ is the charge contributed to atom $\Omega$ by the bond to atom $\Omega^{\prime}, \mathbf{R}_{\Omega}$ is the position vector of atom $\Omega$ with respect to an arbitrary origin, and $\mathbf{R}_{\mathrm{b}}\left(\Omega \mid \Omega^{\prime \prime}\right)$ is the positional vector of the bond critical point between atoms $\Omega$ and $\Omega^{\prime}$. The first part of equation (3) describes the atomic polarization $\mu_{\mathrm{p}}(\Omega)$ term, whereas the second part is the charge translation $\mu_{\mathrm{c}}(\Omega)$ component. Equation (3) can then be rewritten as

$$
\mu(\Omega)=\mu_{\mathrm{p}}(\Omega)+\mu_{\mathrm{c}}(\Omega)
$$

The atomic polarization term comes from the integration of the dipolar density function within the atomic basin, and the charge translation term is given by the weighted sum of atomic charge translations from the atom's nucleus to each bond critical point (BCP) connected to that atom. The sign and value of the charge translation component strongly depend on the nature and number of the groups bonded to the selected atom. The atomic polarization is taken directly from partitioning of electron density, whereas the charge translation component is computed by adopting some constraints. First of all, the sum of net atomic charges must be equal to the molecular charge, which in the simplest case is equal to zero. Each atomic charge $Q(\Omega)$ is expressed by the sum of bond charges $Q\left(\Omega \mid \Omega^{\prime}\right)$ :

$$
Q(\Omega)=\sum_{\Omega^{\prime} \neq \Omega}^{N_{a}} Q\left(\Omega \mid \Omega^{\prime}\right),
$$

where $Q\left(\Omega \mid \Omega^{\prime}\right)=0$ if $\Omega$ and $\Omega^{\prime}$ are not bonded. Furthermore, for each bond

$$
Q\left(\Omega \mid \Omega^{\prime}\right)+Q\left(\Omega^{\prime} \mid \Omega\right)=0 .
$$

If a ring $R$ is present in the system, then the sum of bond charges within the ring is equal to zero:

$$
\sum_{\Omega \in R} Q(\Omega \mid \Omega+1)=0
$$

where $\Omega$ is an atom belonging to the ring $R$. To avoid drastic changes of the shape and values of atomic polarizabilities caused by the presence of weak intra- and intermolecular interactions, PolaBer uses a weighting scheme that is inversely proportional to the bond strength measured, for example, by the electron density at the BCP:

$$
\lambda(\Omega \mid \Omega+1)=1 / \rho(\Omega \mid \Omega+1) .
$$

Thus equation (7) is rewritten as

$$
\sum_{\Omega \in R} \lambda(\Omega \mid \Omega+1) Q(\Omega \mid \Omega+1)=0 .
$$

If no ring is present in a system, equations (5) and (6) produce $\left(N_{\mathrm{a}}+\right.$ $\left.N_{\mathrm{b}}-1\right)$ linearly independent equations $\left(N_{\mathrm{a}}\right.$ and $N_{\mathrm{b}}$ being the number of atoms and number of bond critical points, respectively). The ring conditions add as many independent equations as rings found in the structure. After solving the set of equations, we obtain bond charges that are then used to calculate the charge translation terms of the atomic dipole moments and thus the total atomic dipole moments.

Atomic polarizabilities are numerical derivatives of the corresponding atomic dipoles with respect to the applied field. Calculations are carried out numerically, and thus

$$
\alpha_{i j}(\Omega)=\frac{\mu_{i}^{\varepsilon_{j}}(\Omega)-\mu_{i}^{0}(\Omega)}{\varepsilon_{j}},
$$

where $\mu_{i}^{\varepsilon_{j}}(\Omega)$ is the atomic dipolar component along the $i$ direction computed with a given electric field $(0$ or $\varepsilon)$ in direction $j$. In general, PolaBer uses calculations at $\pm \varepsilon_{j}$ and computes derivatives by averaging two dipole differences. Moreover, $\varepsilon$ is a sufficiently small electric field (typically 0.005 a.u.) to guarantee a better extraction of the linear component of the electron polarization. Because the coupling of atomic volumes and atomic charges is not taken into account, the so-obtained atomic polarizability tensors may be nonsymmetric (whereas, by definition, polarizability is a symmetric tensor). This problem, however, can be circumvented by decomposing the polarizability tensor into symmetric and antisymmetric terms as recommended by Nye (1985). This procedure reconstructs 
very accurately the total molecular polarizability (which is comparable to the molecular polarizability derived from the analytical double derivative of the energy with respect to the field). In fact, antisymmetric components of atomic polarizabilities cancel each other in a molecular summation.

\section{Program description}

PolaBer is written in the Fortran90 programming language. It consists of a main program and a number of subroutines. The program requires atomic charges, polarization dipoles and critical points of the electron density calculated from external programs, with and without an applied electric field. PolaBer uses these entries to calculate the bond charge translation terms defined in equation (4) and the derivatives of the dipolar terms with respect to the field, and hence the atomic polarizability tensors, which are eventually symmetrized. If a unit cell is given, the program will also calculate the crystal refractive indices.

The Windows version of the program is available from http:// www.macchi.dcb.unibe.ch/PolaBer.html. Source code and compiled executables for other platforms are available on request from the authors. A manual with all the functions and commands in the program is attached to the source code. The main code is supported by a visualization tool, ViewTensor, which generates an X3D file representing data in three dimensions. It is based on view3dscene by Kamburelis (2011). PolaBer can be run either on Linux or on Windows platforms with the use of the ViewTensor tool. A screenshot of ViewTensor is given in Fig. 1.

\subsection{Calculation options}

PolaBer requires the electron density partitioning of an unperturbed and field-perturbed molecular system. In principle this step is completely independent, meaning that the electron density could be obtained from calculations as well as from experiments, with whatsoever kind of approximations. In practice, molecular orbital wavefunctions and corresponding electron density partitioning are the most immediate choices. Since polarizabilities obtained with PolaBer

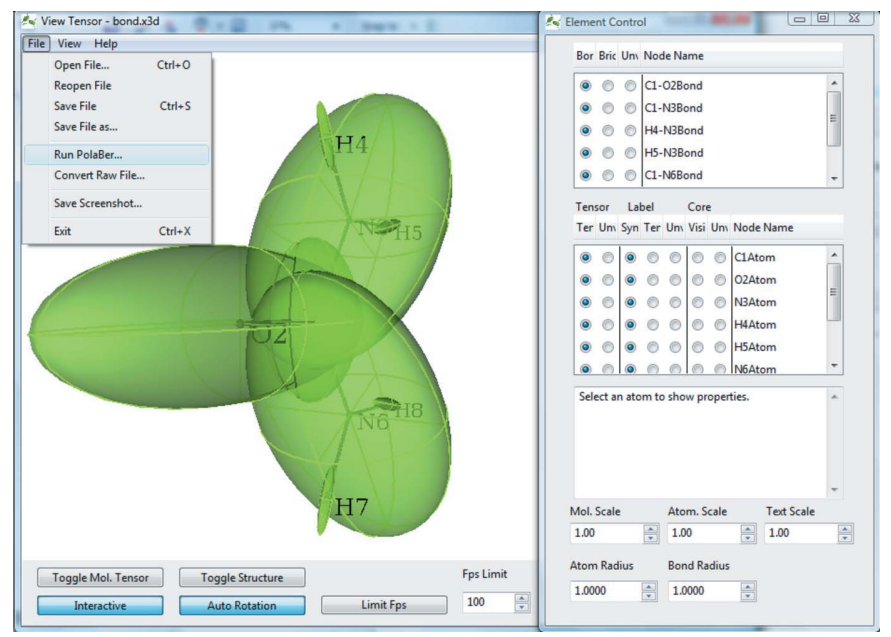

Figure 1

Graphical user interface for visualization of atomic polarizabilities and running PolaBer (available only on Windows platforms). are numerical derivatives of the corresponding atomic dipoles with respect to the applied electric field, calculations of wavefunctions should be carried out at zero electric field as well as under a small electric field, e.g. 0.005 a.u. directed along $\pm \mathbf{X}, \pm \mathbf{Y}$ and $\pm \mathbf{Z}$. The value of the proposed electric field was proven (Krawczuk et al., 2011) to be sufficiently small to obtain good numerical derivatives of the dipolar density, except for some systems which required a smaller field of 0.001 a.u. for more precise evaluation of the atomic polarizabilities. After a set of wavefunctions has been obtained for a given system, integration of the electron density is needed. This is done by performing QTAIM partitioning with the use of AIMAll or XD2006 (Volkov et al., 2006), after expanding the molecular density in terms of multipoles. Other software could be used, of course, but is not directly interfaced at the moment, and the output of any alternative software would require processing in order to provide PolaBer with the required information. For this reason a generalized input file is also accepted (which can be written by any external software). The format and input requirements are given in Table 1. A schematic representation of the computational steps that need to be taken before entering the PolaBer routine is given in Fig. 2. An example of a jobfile for launching all calculations, as well as an example of a Gaussian input file, is included in the supporting materials. ${ }^{1}$ PolaBer starts with reading and importing necessary data from density integration. The following data are read from AIMAll/XD files:

(a) Atomic charges $q(\Omega)$ and coordinates in the Cartesian system

(b) Coordinates of BCPs and values of electron density and Laplacian on BCPs

(c) The atomic polarization component of the atomic dipole moment $\mu_{\mathrm{p}}(\Omega)$

Apart from the AIMAll/XD output files, an additional control file is needed (dipolar.inp), which contains instructions and additional information necessary for PolaBer (see Table 2).

After gathering the necessary data, PolaBer starts the calculations. These are quite rapid. The first step of the procedure is the calculation of the bond charges $Q\left(\Omega \mid \Omega^{\prime}\right)$, necessary to obtain the charge translation components of the atomic dipole moments. In this step PolaBer also searches for rings and cages within the molecular system and applies a weighting scheme (see previous section), if requested by the user.

In the next step, the total atomic dipole moments are calculated at each electric field. The atomic polarizability tensors are then obtained after symmetrization of the tensor.

\footnotetext{
${ }^{1}$ Supplementary data for this paper are available from the IUCr electronic archives (Reference: TO5075).
} 
Table 2

Layout of the dipolar.inp file.

\begin{tabular}{|c|c|}
\hline \multirow[t]{3}{*}{ INTEG WEIGHT VERBOSE $\mathrm{n}$} & INTEG: specifies which program was used for integration of electron density (currently available $A I M A l l / X D$ ) \\
\hline & WEIGHT: if present specifies the use of a weighting scheme (see text for more details) \\
\hline & VERBOSE $\mathrm{n}(\mathrm{n}=0,1,2)$ : setting $\mathrm{n}$ greater than 0 switches on extra printout to the bond. out file \\
\hline \multirow[t]{2}{*}{ nfiles } & nfiles: number of files to be read into PolaBer from AIMAll/XD: \\
\hline & $\begin{array}{l}\text { nfiles }=4 \text { if calculations were done for EFIELD }=0, X, Y, Z \\
\text { nfiles }=7 \text { if calculations were done for EFIELD }=0, \pm X, \pm Y, \pm Z \text { (highly recommended) }\end{array}$ \\
\hline fname1 EFIELD EFX EFY EFZ & fname: list of all $A I M A I l / X D$ file names (*.sum, *. out) with specification of applied electric field (EFFIELD), i.e. xp. sum \\
\hline fname2 EFIELD EFX EFY EFZ & $\begin{array}{l}\text { EFIELD } 0.005 \text { 0. } 0 \text {. specifies output from AIMAll and an applied electric field in the } \mathbf{X} \text { direction with the value of } \\
0.005 \text { a.u. }\end{array}$ \\
\hline cell parameters & Cell parameters given in $\AA$ in fractional coordinates with s.u. values \\
\hline 10.000010 .000010 .000090 .00090 .00090 .000 & \\
\hline $0.00000 .0000 \quad 0.0000 \quad 0.0000 .0000 .000$ & \\
\hline LATT N & $\begin{array}{l}\text { Lattice type, according with } S H E L X \text { convention: } 1=P, 2=I, 3=R_{\text {obv }} \text { on hexagonal axes, } 4=F, 5=A, 6=B, 7=C \text {, negative } \\
\text { sign indicates noncentrosymmetric structure }\end{array}$ \\
\hline SYMM $\mathrm{x} 1 \mathrm{y} 1 \mathrm{z} 1 \mathrm{x} 2 \mathrm{y} 2 \mathrm{z} 2 \mathrm{x} 3 \mathrm{y} 3 \mathrm{z} 3$, tx ty tz & $\begin{array}{l}\text { Symmetry operations within a given space group } \\
\mathrm{x} 1, \mathrm{y} 1, \mathrm{z} 1 \text { : first row of rotation matrix; } \mathrm{x} 2, \mathrm{y} 2, \mathrm{z} 2 \text { : second row of rotation matrix; } \mathrm{x} 3, \mathrm{y} 3, \mathrm{z} 3 \text { : third row of rotation matrix } \\
\mathrm{tx}, \mathrm{ty}, \mathrm{tz} \text { : translational components in decimal notation, } \pm 0.25,0.33,0.5,0.67,0.75\end{array}$ \\
\hline TYPE ntype & Number of atom types \\
\hline SFAC C H O & Scattering factors for each type of atom \\
\hline UNIT $10 \quad 1010$ & Number of atoms of each type in a unit cell, in SFAC order \\
\hline LAMBDA 0.71073 & Wavelength in $\AA$ \\
\hline ATOMLIST & Creates a summary table for a chosen fragment \\
\hline nlist & Number of atoms to be included \\
\hline at1 at $2 \ldots$ & List of atoms building a chosen fragment for which a summary table will be created in bond.out \\
\hline
\end{tabular}

PolaBer calculates also an estimation of the bond polarizability:

$$
\boldsymbol{\alpha}_{\Omega-\Omega^{\prime}}=\mathbf{r}_{\Omega \Omega^{\prime}}^{\mathrm{T}}\left(\boldsymbol{\alpha}_{\Omega}+\boldsymbol{a}_{\Omega^{\prime}}\right) \mathbf{r}_{\Omega \Omega^{\prime}},
$$

where $\mathbf{r}_{\Omega \Omega^{\prime}}$ is a unit vector in the direction of the $\Omega-\Omega^{\prime}$ bond. Therefore, the bond polarizability is a projection of the atomic polarizability tensor along the bond. This definition is 'exact', in the sense that the bond polarizability is not a fitted quantity but it comes univocally from the calculated distributed atomic polarizabilities of the system. On the other hand, it is important to stress that in fact the concept of bond polarizability lacks a precise definition. Therefore, $\alpha_{\Omega-\Omega^{\prime}}$ cannot be compared with other bond polarizabilities proposed in the literature, because it is based on different definitions.

\subsection{Output files}

Currently, the program prints out the following output files:

(i) bond.out - a common format file containing a summary of atomic/bond polarizability calculations.

(ii) bond.res - a crystallographic format containing fractional coordinates of a studied system; the format is the same as the one produced by the SHELX program (Sheldrick, 2008). Instead of

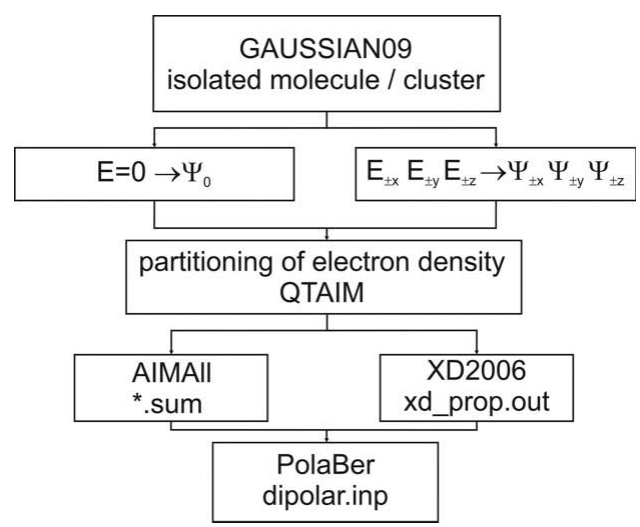

Figure 2

Schematic representation of necessary steps before entering PolaBer. $E_{i}$ - applied electric field in a given direction; $\Psi_{i}$ - wavefunction after applying an external electric field. atomic displacement parameters, components of atomic polarizability tensors are given, scaled by a factor of 0.1 .

(iii) bond.x3d - a file representing data as a three-dimensional view which is visualized with the locally developed ViewTensor program.

Calculated quantities in the output file are summarized in two main tables: atomic and bond properties. Components of atomic polarizability tensors are listed in Cartesian coordinates as well as in the crystal system defined in the input file (dipolar.inp). A summary of the main quantities printed out in the bond.out file is given in the supplementary materials in Table S1.

\section{Applications}

\subsection{Isolated molecules}

To illustrate how PolaBer works, we start with calculations on isolated molecules of urea and L-alanine. In order to obtain wavefunctions for both molecules, $a b$ initio calculations were carried out with the Gaussian09 package at the B3LYP/6-311++G(2d,2p) level. The geometry of urea was optimized, whereas for the zwitterionic form of L-alanine coordinates were taken from neutron diffraction data (Lehmann et al., 1972) and kept frozen for further calculations. These geometries were then used to calculate the wavefunctions under electric field perturbation, using of course the same level of theory. Topological analysis and integration of the electron density in the atomic basins were carried out using AIMAll.
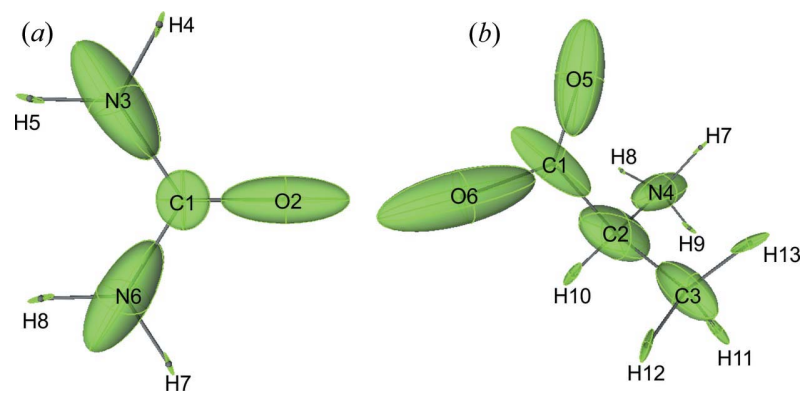

Figure 3

Graphical representation of distributed atomic polarizabilities for $(a)$ urea and $(b)$ L-alanine molecules. The scaling factor for atomic polarizabilities is $0.4 \AA^{-2}$. 
Table 3

Components of atomic polarizability tensors of urea calculated with QTAIM partitioning based on $\mathrm{B} 3 \mathrm{LYP} / 6-311++\mathrm{G}(2 d, 2 p)$ calculations.

All quantities are in atomic units $\left(\mathrm{Bohr}^{3}\right)$. Mol refers to the total molecular polarizability tensor.

\begin{tabular}{llrrllr}
\hline Atom & $\alpha_{11}$ & \multicolumn{1}{c}{$\alpha_{22}$} & $\alpha_{33}$ & $\alpha_{12}$ & $\alpha_{13}$ & \multicolumn{1}{c}{$\alpha_{23}$} \\
\hline C1 & 2.20 & 5.98 & 5.32 & 0 & 0 & 0.00 \\
O2 & 4.85 & 4.72 & 12.89 & 0 & 0 & 0.00 \\
N3 & 8.20 & 10.94 & 7.33 & 0 & 0 & -3.36 \\
H4 & 0.48 & 2.27 & 0.70 & 0 & 0 & 0.66 \\
H5 & 0.52 & 0.66 & 2.60 & 0 & 0 & -0.51 \\
N6 & 8.20 & 10.94 & 7.33 & 0 & 0 & 3.36 \\
H7 & 0.48 & 2.27 & 0.70 & 0 & 0 & -0.66 \\
H8 & 0.52 & 0.66 & 2.60 & 0 & 0 & 0.51 \\
& & & & & & 0 \\
Mol & 25.43 & 38.44 & 39.48 & 0 & 0 & 0 \\
\hline
\end{tabular}

Graphical representations of the calculated atomic polarizabilities for urea and L-alanine molecules are given in Fig. 3. The analysis of both images indicates a number of features that are characteristic of atomic polarizabilities. First of all the electron density polarization strongly affects the polarizability. This is evident especially in the direction of stronger chemical bonds. In fact, polarizability ellipsoids are strongly elongated towards more polarizable atoms (see the $\mathrm{C}=\mathrm{O}$ and $\mathrm{C}-\mathrm{N}$ bonds), and the higher the difference of electronegativity between bonded atoms, the more prolate the polarizability ellipsoids along the bond. In the case of $\mathrm{H}$ atoms, the values of the polarizability tensors are very small compared to those of non- $\mathrm{H}$
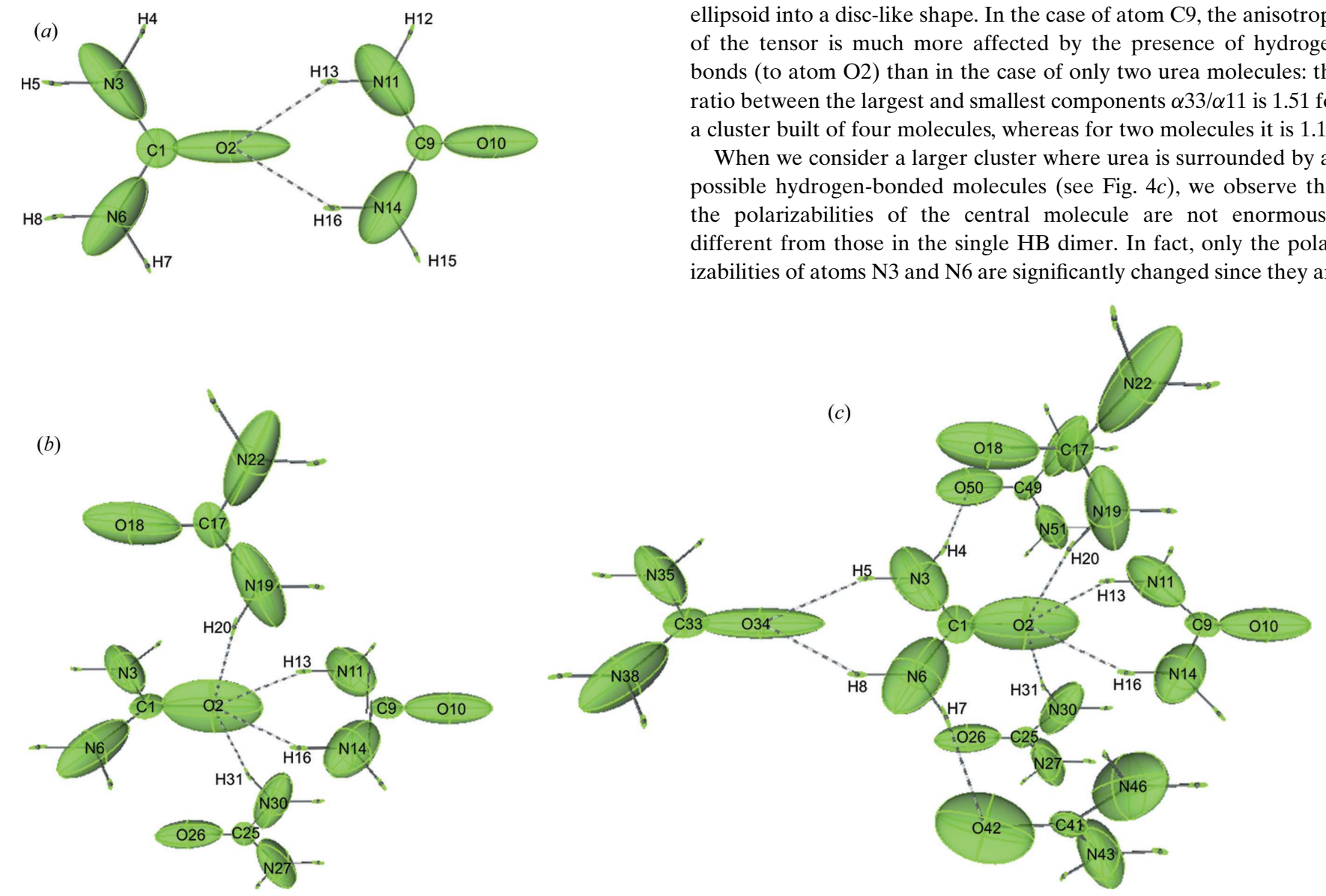

Figure 4

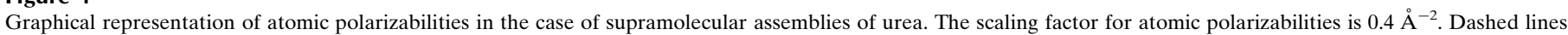
indicate hydrogen bonds between molecules. atoms (see any $\mathrm{H}-X$ bond), which is due to the small electronic population of $\mathrm{H}$ atoms. Nevertheless, it is quite clear that the largest component of the polarizability tensor of any $\mathrm{H}$ atom is in the direction of an $\mathrm{H}-X$ bond. For urea, the values of the atomic/ molecular polarizabilities obtained with PolaBer are summarized in Table 3. Note that the polarizability tensors fulfill the symmetry requirements of the molecule.

\subsection{Supramolecular assemblies}

We now analyze the influence of interatomic interactions on the shape and orientation of polarizability tensors. Let us first consider two molecules of urea interacting through $\mathrm{N}-\mathrm{H} \cdots \mathrm{O}$ hydrogen bonds (HBs). From Fig. 4(a), we can see that the ellipsoid representing the polarizability tensor of atom $\mathrm{O} 2$ (acceptor of both $\mathrm{HBs}$ ) is modified because of the hydrogen bonds (compare with Fig. 3a). Since HBs are symmetrically equivalent, the pronounced elongation of the oxygen ellipsoid is directed along the bisector of the N11C9-N14 angle towards the C9-O10 carbonyl group. When $\mathrm{H}$ atoms are involved in HBs, the orientation of their ellipsoids changes (with respect to the isolated molecule), towards the acceptor of both hydrogen bonds.

If urea is surrounded by three other molecules and atom $\mathrm{O} 2$ is involved in four instead of two hydrogen bonds of $\mathrm{N}-\mathrm{H} \cdots \mathrm{O}$ type (Fig. $4 b$ ), the oxygen polarizability tensor behaves differently. While the elongation of the ellipsoid towards the $\mathrm{C} 9-\mathrm{O} 10$ bond is comparable to the previous example, the presence of two additional $\mathrm{HBs}$ in a plane perpendicular to urea $(\mathrm{C} 1-\mathrm{O} 2$ bond) flattens the ellipsoid into a disc-like shape. In the case of atom $\mathrm{C}$, the anisotropy of the tensor is much more affected by the presence of hydrogen bonds (to atom $\mathrm{O} 2$ ) than in the case of only two urea molecules: the ratio between the largest and smallest components $\alpha 33 / \alpha 11$ is 1.51 for a cluster built of four molecules, whereas for two molecules it is 1.12.

When we consider a larger cluster where urea is surrounded by all possible hydrogen-bonded molecules (see Fig. $4 c$ ), we observe that the polarizabilities of the central molecule are not enormously different from those in the single HB dimer. In fact, only the polarizabilities of atoms N3 and N6 are significantly changed since they are 


\section{computer programs}

additionally involved in two hydrogen bonds with atom O34. What is more interesting in this example is how the direction and strength of the hydrogen bond affect the shape and orientation of ellipsoids representing the polarizability tensors of $\mathrm{O}$ atoms. For example, if we consider atom $\mathrm{O} 42$, the presence of an $\mathrm{HB}$ towards atom $\mathrm{H} 7$ rotates the ellipsoid in the direction of the bond and makes it less elongated along the $\mathrm{C}-\mathrm{O}$ bond compared to the isolated state. Conversely, atom $\mathrm{O} 34$ becomes more anisotropic along the $\mathrm{C}-\mathrm{O}$ bond owing to the formation of two symmetrically equivalent hydrogen bonds, whereas atom $\mathrm{O} 10$ behaves like the atom from the isolated molecule since it is not involved in any intermolecular interactions.

\subsection{Crystals}

One of the main applications of distributed atomic polarizabilities is the estimation of crystal optical properties. The crystal dielectric constant can be calculated from the atomic polarizabilities. In first approximation, this could be calculated as simply the sum of unperturbed polarizabilities calculated in the gas phase. However, this would highly underestimate the dielectric tensor, ignoring the enhancement of the polarizability in the field generated by other molecules. The corresponding crystal refractive indices would also be underestimated. The advantage of distributed atomic polarizabilities is that one can consider a molecule embedded in a cluster of surrounding molecules, as described in the previous section, then easily extract the polarizability of just the central molecule (taking advantage of the atomic distribution) and eventually use this central polarizability to calculate the crystal properties. This approach enables to us account at least for the perturbation of the first coordination sphere, which may be more than just a classical electrostatic interaction, especially for crystals with a strong hydrogen-bond network. A second correction would be then to account for the longrange interactions, which can be safely approximated with a simple electrostatic perturbation and treated in classical terms, basically applying the Clausius Mossiotti theory or the anisotropic Lorentz field-factor approach. A more comprehensive theory for this approach was provided by Dunmur (1972), Cummins et al. (1976) and Bounds \& Munn (1981). Jayatilaka et al. (2009) and Whitten et al. (2006) have shown the advantages of this method, testing molecular polarizabilities calculated at different quantum mechanical levels, including the X-ray constrained wavefunction. Again there could be an advantage to using the distributed atomic polarizabilities: in fact it was noted that a perturbation calculated to a 'central' polarizability was not adequate, in particular if the molecule and the crystal packing are quite anisotropic. For this reason, Bounds \& Munn (1977) suggested to 'distribute' the molecular polarizability of the central molecule on different sites, thus enabling a more accurate description. However, the distribution proposed was a simple equi-partition of the molecular tensor on some atomic sites, whereas it is clear from our examples that the atomic polarizabilities are quite diverse and the approach of Bounds and Munn, albeit more precise than a central polarizability approach, may not be sufficiently accurate. Having, instead, the exact atomic polarizabilities, it is possible to perform the calculation with much more precision, using the same formalism as Bounds \& Munn (1977).

In Table 4, we report the calculated refractive indices of urea based on $(a)$ the isolated molecular polarizability (here the distributed atomic polarizability is not used), (b) the polarizability of a molecule extracted from the first coordination sphere and $(c)$ the molecular polarizability computed in a polarizable continuum medium, mimicking the crystal dielectric constant. Calculations $(a)$ can be correct if the interaction of distant molecules is evaluated using
Table 4

Dielectric susceptibility and refractive indices for urea.

Both quantities are dimensionless. Note that the crystal class of urea implies $\chi_{i j}=0$ for $i \neq$ $j$ and $\chi_{11}=\chi_{22}$. Subscripts o and e refer to ordinary and extraordinary rays, respectively.

\begin{tabular}{lllll}
\hline & $\chi_{11}$ & $\chi_{33}$ & $n_{\mathrm{o}}$ & $n_{\mathrm{e}}$ \\
\hline $\begin{array}{l}\text { (a) Sum of gas phase molecular polarizabilities } \\
\quad \text { anisotropic Lorentz correction }\end{array}$ & 0.821 & 1.012 & 1.349 & 1.418 \\
$\quad$ + rigorous local field Lorentz correction & 1.131 & 1.526 & 1.460 & 1.589 \\
$\quad$ + rigorous local field Lorentz correction on & 1.118 & 1.571 & 1.456 & 1.603 \\
$\quad \begin{array}{l}\text { distributed atomic polarizabilities } \\
\text { (b) Sum of molecular polarizabilities from cluster }\end{array}$ & 0.943 & 1.578 & 1.455 & 1.605 \\
$\quad$ calculation & & 1.207 & 1.394 & 1.486 \\
$\begin{array}{l}\text { (c) Molecule in a polarizing continuum medium } \\
\text { (d) Extrapolated to infinite wavelength } \dagger\end{array}$ & 1.170 & 1.510 & 1.473 & 1.584 \\
\hline & - & - & 1.47 & 1.58 \\
\hline
\end{tabular}

$\dagger$ Halbout et al. (1979).

Munn's schemes, namely the so-called Lorentz anisotropic approximation or the rigorous local-field approximation (calculated using molecular or distributed atomic polarizabilities). It is notable that the latter approach enables a calculation that is very close to the periodic $a b$ initio methods as well as the experimental values extrapolated to infinite wavelength (Halbout et al., 1979) (see Table 4). Combination of the molecule in a cluster and the Lorentz approaches is not sensible because it would lead to an overcorrection (first coordination sphere counted twice) and larger refractive indices. Through the example of Table 4, we just want to illustrate how much of the total enhancement of the dielectric constant is actually due to the first coordination sphere and how much is instead coming from the longrange interaction. Indeed, the second part is in fact dominating.

There is an additional calculation shown in Table 4, which is also worthy of comment: the molecular wavefunction calculated using a dipolar continuum medium, an approach normally adopted in order to simulate the effect of a solvent $(c)$. If the dielectric constant computed for an isolated molecule of urea is used (assuming the same molecular volume as in the crystal), the corresponding crystal refractive indices match almost exactly the experimental ones, without further corrections. This observation is very interesting, because the calculation of distributed polarizabilities for a single molecule of urea within the polarizable medium is just marginally more expensive than the calculation in the absence of such a medium and definitely less time consuming than the calculation of a cluster or the fully periodic system. Of course such a calculation does not require Lorentz correction, because this is implicitly accounted for.

\section{Conclusions}

In this paper, we have reported on a new routine that enables the calculation of distributed atomic polarizabilities from molecular orbital wavefunctions (or in principle also multipolar expansions) of the electron density distribution of the ground state and the electric field perturbed electron density of a molecule, or in principle of a crystal as well. The program uses electron density partitioning to define the atomic boundaries. In the illustrated examples, the partitioning is obtained through the QTAIM approach, but other schemes would be equally suitable and will be tested in the future.

One important use of this approach is the calculation of optical properties in crystals, which requires the evaluation of accurate molecular polarizabilities but also of supramolecular perturbations, due to short-range interactions within the first coordination sphere as well as to long-range electrostatic perturbations. The calculation of the refractive indices of a crystal is straightforward, and in future 
work we will also show the evaluation of optical activity and some nonlinear optical properties, by extending this approach to hyperpolarizabilities.

Other applications of this method, also useful for crystallographic applications, concern the evaluation of intermolecular induction and dispersion energy terms, within the semi-classical approaches of intermolecular interactions.

PolaBer will be developed in the future having these applications in mind.

This work was supported by the Swiss National Science Foundation (project No. 141271) and by the Swiss Conference of Rectors (CRUS). We thank Mr Leonardo dos Santos for testing the program and suggesting improvements.

\section{References}

Bader, R. F. W. (1989). J. Chem. Phys. 91, 6989-7001.

Bader, R., Keith, T., Gough, K. \& Laidig, K. (1992). Mol. Phys. 75, 1167-1189.

Bader, R. F. W., Larouche, A., Gatti, C., Carroll, M. T., MacDougall, P. J. \& Wiberg, K. B. (1987). J. Chem. Phys. 87, 1142-1152.

Bounds, P. J. \& Munn, R. W. (1977). Chem. Phys. 24, 343-353.

Bounds, P. J. \& Munn, R. W. (1981). Chem. Phys. 59, 47-53.

Cummins, P. G., Dunmur, D. A., Munn, R. W. \& Newham, R. J. (1976). Acta Cryst. A32, 847-853.

Devarajan, V. \& Glazer, A. M. (1986). Acta Cryst. A42, 560-569.

Dittrich, B., Hübschle, C. B., Luger, P. \& Spackman, M. A. (2006). Acta Cryst. D62, 1325-1335.

Dunmur, D. (1972). Mol. Phys. 23, 109-115.

Glazer, A. M. \& Stadnicka, K. (1986). J. Appl. Cryst. 19, 108-122.

Gough, K. M., Yacowar, M. M., Cleve, R. H. \& Dwyer, J. R. (1996). Can. J. Chem. 74, 1139-1144.

Halbout, J. M., Blit, S., Donaldson, W. \& Tang, C. L. (1979). IEEE J. Quantum Electron. 15, 1176-1180.
Hansen, N. K. \& Coppens, P. (1978). Acta Cryst. A34, 909-921.

Hättig, C., Jansen, G., Heß, B. A. \& Ángyán, J. G. (1996). Can. J. Chem. 74 976-987.

Hirshfeld, F. L. (1977). Isr. J. Chem. 16, 198-201.

Jayatilaka, D., Munshi, P., Turner, M. J., Howard, J. A. \& Spackman, M. A. (2009). Phys. Chem. Chem. Phys. 11, 7209-7218.

Kamburelis, M. (2011). view3dscene (Version 3.10.1), http://castle-engine. sourceforge.net.

Keith, T. A. (2007). The Quantum Theory of Atoms in Molecules: From Solid State to DNA and Drug Design, edited by C. F. Matta \& R. J. Boyd, ch. 3. Weinheim: Viley-VCH.

Keith, T. A. (2013). AIMAll. Version 13.05.06. TK Gristmill Software, Overland Park KS, USA.

Krawczuk, A., Pérez, D., Stadnicka, K. \& Macchi, P. (2011). Trans. Am. Crystallogr. Assoc. pp. 1-25.

Kurki-Suonio, K. (1968). Acta Cryst. A24, 379-390.

Kurki-Suonio, K. (1977). Isr. J. Chem. 16, 132-136.

Laidig, K. E. \& Bader, R. F. W. (1990). J. Chem. Phys. 93, 7213-7224.

Lehmann, M. S., Koetzle, T. F. \& Hamilton, W. C. (1972). J. Am. Chem. Soc. 94, 2657-2660.

Le Sueur, C. R. \& Stone, A. J. (1993). Mol. Phys. 78, 1267-1291.

Nye, J. F. (1985). Physical Properties of Crystals: Their Representation by Tensors and Matrices. Oxford University Press.

Pichon-Pesme, V., Lecomte, C. \& Lachekar, H. (1995). J. Phys. Chem. 99, 6242-6250.

Sheldrick, G. M. (2008). Acta Cryst. A64, 112-122.

Stewart, R. F. (1976). Acta Cryst. A32, 565-574.

Stewart, R. F., Bentley, J. \& Goodman, B. (1975). J. Chem. Phys. 63, 3786.

Stone, A. (1985). Mol. Phys. 56, 1065-1082.

Volkov, A., Li, X., Koritsanszky, T. S. \& Coppens, P. (2004). J. Phys. Chem. A, 108, 4283-4300.

Volkov, A., Macchi, P., Farrugia, L. J., Gatti, C., Mallinson, P., Richter, T. \& Koritsanszky, T. (2006). XD2006, http://xd.chem.buffalo.edu/.

Whitten, A. E., Jayatilaka, D. \& Spackman, M. A. (2006). J. Chem. Phys. 125, 174505 .

Zarychta, B., Pichon-Pesme, V., Guillot, B., Lecomte, C. \& Jelsch, C. (2007). Acta Cryst. A63, 108-125. 